\title{
JUICIOS EN LA PREPARACIÓN DE ESTADOS FINANCIEROS DE EMPRESAS MINERAS PERUANAS
}

\author{
JUDGMENTS IN PREPARATION OF FINANCIAL STATEMENTS OF PERUVIAN \\ MINING COMPANIES
}

Rosa Milagros Castañeda Moreano Pontificia Universidad Católica del Perú Lima, Perú

ORCID: https://orcid.org/0000-0003-3097-8504

Correo electrónico: rcastanedamoreano@gmail.com

\section{RESUMEN}

Objetivo: Identificar si existe una revelación diferenciada de los juicios de las empresas mineras peruanas para el año 2020 en relación con las Normas Internacionales de Información Financiera (NIIF), y describir si se presenta un suficiente nivel de detalle de los supuestos asumidos en las empresas mineras peruanas en el año 2020 en relación con las NIIF. Método: Enfoque cualitativo, parcialmente descriptivo, no experimental, transversal y un análisis de contenido del marco normativo de las NIIF y de los estados financieros de las empresas mineras peruanas. Resultados: Cuatro de las once empresas mineras peruanas no efectúan una revelación diferenciada de los juicios y, por otro lado, los supuestos asumidos son genéricos y no proporcionan mayor detalle. Conclusión: Existe una apertura de criterios en la revelación de juicios y una carencia de revelación suficiente de los supuestos asumidos.

Palabras clave: Juicios; políticas contables; estimaciones contables críticas; supuestos; incertidumbre.

\begin{abstract}
Objective: Identify if there is a differentiated disclosure of the Peruvian mining companies' judgments for the year 2020 in relation to the International Financial Reporting Standards (IFRS), as well as describe if there is a sufficient level of detail of the presumed assumptions in the Peruvian mining companies in 2020 in relation to the IFRS. Method: Qualitative, partially descriptive, non-experimental, transversal approach and a content analysis of the normative framework of the IFRS and the financial statements of Peruvian mining companies. Results: Four out of the eleven Peruvian mining companies do not make a differentiated disclosure of the lawsuits, and, on the other hand, the presumed assumptions are generic and do not provide greater detail. Conclusion: There is an opening of criteria in the disclosure of judgments and a lack of sufficient disclosure of the presumed assumptions.
\end{abstract}

Keywords: Judgements; accounting policies; critical accounting estimates; assumptions; uncertainty.

(c) Los autores. Este artículo es publicado por la revista Quipukamayoc de la Facultad de Ciencias Contables, Universidad Nacional Mayor de San Marcos. Este es un artículo de acceso abierto, distribuido bajo los términos de la licencia Creative Commons Atribución 4.0 Internacional (CC BY 4.0) [https://creativecommons.org/licenses/ by/4.0/deed.es] que permite el uso, distribución y reproducción en cualquier medio, siempre que la obra original sea debidamente citada de su fuente original. 


\section{INTRODUCCIÓN}

Los juicios en la preparación de estados financieros toman relevancia puesto que requieren el criterio de la gerencia y una adecuada revelación. La información revelada, en el mejor de los casos, incluye una estimación -hecho que abre la discusión sobre los criterios de interpretación-, el nivel de detalle de la revelación y los efectos sobre el valor de las empresas y sobre las decisiones de dicha información.

Respecto de la información cuantitativa y cualitativa que presenta una empresa en sus estados financieros, Hendriksen y van Breda (1992) sostienen que una adecuada revelación de información cualitativa es necesaria por la dificultad que se presenta al evaluar su materialidad y relevancia, debido a que los usuarios asumen ciertos supuestos y le asignan diferente ponderación al evaluar dicha información, "por lo tanto, se debe buscar el punto donde se puede decir que la información es lo suficientemente importante en la toma de decisiones como para no omitirla" (p. 859).

Por otro lado, los usuarios prefieren la información cuantitativa, pues la ausencia de esta puede generar costos de interpretación y procesamiento, debido a los supuestos a asumir y al bajo nivel de confiabilidad que se podría obtener (Michels, 2017). En ese sentido, los usuarios se ven en la necesidad de evaluar diversos escenarios con base en la información disponible.

Siendo así, el marco normativo de las Normas Internacionales de Información Financiera (NIIF) considerado para la presente investigación, comprende la NIC 1 Presentación de Estados Financieros en lo concerniente a los supuestos asumidos respecto de la naturaleza, impacto y resolución de la incertidumbre, y la NIC 8 Políticas Contables, Cambios en las Estimaciones Contables y Errores en cuanto al uso del juicio frente a los cambios en las estimaciones contables, así como el Marco Conceptual para la Información Financiera, con un énfasis en la consideración de juicios, estimaciones y modelos para la preparación de estados financieros. A continuación, se presenta un resumen de cada uno de estos.

El Marco Conceptual para la Información Financiera (MC), en relación con el reconocimiento, destaca la vinculación entre la estimación y la representación fiel, constituyéndose un aspecto de importancia (International Financial Reporting Standards Foundation [IFRS Foundation], 2020). Es decir, es importante que se consideren los criterios de reconocimiento para que los estados financieros sean relevantes y representen fielmente los hechos económicos.
En lo que respecta a la NIC 1 Presentación de Estados Financieros, existen dos tipos de juicios que requieren ser revelados: los juicios que implican estimaciones y los juicios en la aplicación de las políticas contables (IFRS Foundation, 2020). Esta distinción es necesaria para la interpretación de la información cualitativa revelada por las empresas.

Asimismo, en la NIC 1 se destaca la descripción de las causas de incertidumbre en las estimaciones como aquellas "que tengan un riesgo significativo de ocasionar ajustes significativos en el valor en libros de los activos o pasivos dentro del periodo contable siguiente" (IFRS Foundation, 2020, p. 1256); es decir, indica la necesidad de revelar información sobre los supuestos asumidos en relación con el futuro que se espera ocasionen ajustes significativos revelando una descripción de su naturaleza, importe e impacto sobre los elementos de los estados financieros. Además, la NIC 1 destaca la descripción de dichos supuestos para estimaciones con "mayor dificultad, subjetividad o complejidad en el juicio” (IFRS Foundation, 2020, A1257).

Adicionalmente, la NIC 1 indica la revelación de los juicios sobre las consideraciones del futuro, lo que incluye su naturaleza, nivel de sensibilidad, la definición esperada de la incertidumbre asociada, posibles consecuencias y cambios en los supuestos asumidos siempre que la incertidumbre se mantenga (IFRS Foundation, 2020). Además, cuando la revelación sea impracticable, se indica la necesidad de describir la naturaleza e importe afectado por el supuesto asumido con base en la información disponible (IFRS Foundation, 2020).

Referente a la NIC 8 Políticas Contables, Cambios en las Estimaciones Contables y Errores, esta señala que "los cambios en las estimaciones contables son el resultado de nueva información o nuevos acontecimientos y, en consecuencia, no son correcciones de errores" (IFRS Foundation, 2020, A1317). Por otra parte, la NIC 8 precisa que el juicio aplicado debe decantar en información relevante y fiable, considerando la NIIF relacionada, definiciones y criterios de reconocimiento y medición.

Asimismo, la NIC 8 destaca que "el proceso de estimación implica la utilización de juicios basados en la información fiable disponible más reciente" (IFRS Foundation, 2020, A1327) e involucra aspectos como la valuación por recuperación, valor razonable, vida útil, entre otros. De igual forma, precisa que, en caso surgieran cambios en los supuestos asumidos de las estimaciones, es necesaria su revisión con la información adicional disponible, donde el tratamiento será prospectivo (IFRS Foundation, 2020), lo cual significa que es un proceso dinámico que requiere una continua evaluación. En ese sentido, es importante señalar lo siguiente: 
Un cambio en los criterios de medición aplicados es un cambio en una política contable, y no un cambio en una estimación contable. Cuando sea difícil distinguir entre un cambio de política contable y un cambio en una estimación contable, el cambio se tratará como si fuera un cambio en una estimación contable. (IFRS Foundation, 2020, A1328)

Finalmente, la NIC 8 precisa la revelación de la "naturaleza e importe de cualquier cambio en una estimación contable que haya producido efectos en el periodo corriente, o que se espere vaya a producirlos en periodos futuros, exceptuándose de lo anterior ... cuando fuera impracticable estimar ese efecto" (IFRS Foundation, 2020, A1329), esto es brindar a los usuarios información que permita su adecuada interpretación.

Con lo expuesto, queda claro que el marco normativo contable de las NIIF y el MC distinguen entre juicios para la definición de políticas contables de aquellos juicios en las estimaciones contables críticas, sus revelaciones y potenciales cambios. A partir de ello, se identificaron trabajos anteriores que analizaron el nivel de cumplimiento de dichas revelaciones.

Así, Raubenheimer (2013) identificó los requerimientos de las NIIF sobre la revelación de supuestos e incertidumbres en la estimación en empresas del sector construcción de Sudáfrica, y concluyó que si bien hubo un incremento en los requerimientos de las NIIF referidos a revelaciones de estimaciones contables basados en supuestos, no todas las empresas cumplieron a cabalidad con las revelaciones requeridas.

Por su parte, Chiahemba e Ibiamke (2019) aplicaron una encuesta temática sobre los estados financieros en compañías de bienes de consumo de Nigeria, a fin de identificar su cumplimiento bajo las NIIF respecto de la revelación de juicios críticos y las fuentes de incertidumbre en la estimación. Los autores concluyeron que el nivel de revelación fue menor a lo requerido por las NIIF, por lo que, en general, no proveen suficiente información.

También cabe destacar los hallazgos de Mayorga y Sidhu, quienes identificaron que las compañías australianas públicas presentaron algunas revelaciones sobre supuestos asumidos para estimaciones contables, así como, la publicación de Ernst and Young (2017), en la cual se reportó el rol de la materialidad asociada a la naturaleza e impacto de las estimaciones contables, siendo revelaciones muy generales y carentes de detalle sobre métodos, calidad y variabilidad de los juicios (como se citó en Chiahemba e Ibiamke, 2019).
Adicionalmente, se identificó trabajos que abordaron el impacto de los cambios en las estimaciones contables críticas. Albrecht, Kim y Lee (2017) documentaron la práctica sobre cambios materiales en las estimaciones contables, al igual que los impactos en la evaluación de analistas financieros sobre dicha información. Por otro lado, Glendening (2012) identificó que la relevancia del valor de un rubro de los estados financieros se asocia negativamente con la presencia de la revelación de una estimación contable crítica. Finalmente, Burcă, Nicolăescu y Drăgut (2019) distinguieron las variaciones en las estimaciones contables de aquellas producidas en las políticas contables, donde la ausencia de dicha distinción podría generar un impacto en los modelos de valoración de empresas.

En ese contexto, se busca dar respuesta a las siguientes preguntas: ¿Existe una revelación diferenciada de juicios en las empresas mineras peruanas en el año 2020 en relación con las NIIF? y ¿se presenta un suficiente nivel de detalle de los supuestos asumidos en las empresas mineras peruanas en el año 2020 en relación con las NIIF? A partir de lo anterior, los supuestos hipotéticos son los siguientes: "Las empresas mineras peruanas no revelan de manera diferenciada los juicios para el año 2020 en relación con las NIIF"; y "Las empresas mineras peruanas no presentan un suficiente nivel de detalle acerca de los supuestos asumidos en el año 2020 en relación con las NIIF”.

La investigación se justifica en aspectos prácticos referidos a las diferencias en la revelación de los juicios y sus supuestos asociados, más aún cuando las empresas mineras peruanas reportan información financiera bajo las NIIF desde el año 2011 a la Superintendencia de Mercado de Valores. Mientras que la principal limitación identificada fue la insuficiente revelación de información efectuada por las empresas mineras peruanas.

\section{MATERIAL Y MÉTODOS}

La investigación presentó un enfoque cualitativo, en parte descriptiva, con supuestos hipotéticos en función del análisis de la información obtenida, puesto que no se probaron estadísticamente; asimismo, fue transversal y no experimental, debido a que se obtuvo la información de la plataforma digital de la Superintendencia de Mercado de Valores de Perú con el fin de describir los juicios en la aplicación de las políticas contables y aquellos para las estimaciones contables críticas. La unidad de estudio comprendió los juicios revelados por las empresas mineras peruanas, mientras que la técnica de recolección de datos se centró en el análisis de contenido del marco normativo de las NIIF y los estados financieros de las empresas mineras peruanas por el año 2020 . 
Se seleccionó once empresas mineras peruanas debido a que estas presentan información completa y actualizada de juicios, y su actividad económica es similar entre ellas. Dichas empresas, junto con su nombre abreviado, son las siguientes:

- Compañía de Minas Buenaventura S.A.A. (2020): Buenaventura

- $\quad$ Sociedad Minera Cerro Verde S.A.A. (2020): Cerro Verde

- $\quad$ Sociedad Minera Corona S.A. (2020): Corona

- $\quad$ Sociedad Minera El Brocal S.A.A. (2020): El Brocal

- Minsur S.A. (2020): Minsur

- Nexa Resources Atacocha S.A. (2020): Nexa Atacocha

- $\quad$ Nexa Resources Perú S.A. (2020): Nexa Perú

- Compañía Minera Poderosa S.A. (2020): Poderosa

- Compañía Minera San Ignacio de Morococha S.A.A. (2020): SIMSA

- Southern Perú Copper Corporation, Sucursal del Perú (2020): Southern

- Volcan Compañía Minera S.A.A. (2020): Volcan

\section{RESULTADOS}

Se efectuó el análisis de contenido de los estados financieros de las once empresas mineras peruanas con énfasis en las revelaciones sobre los juicios y los supuestos asumidos. Como parte del análisis realizado, se identificaron (i) los juicios en la aplicación de las políticas contables y (ii) los juicios en las estimaciones contables críticas. En ese sentido, la Tabla 1 presenta la cantidad de conceptos revelados de cada tipo de juicios por el año 2020 de las empresas sujetas a análisis.

Al respecto, se efectuó el análisis de contenido en relación con los objetivos planteados, tomando los estados financieros de cada una de las empresas mineras peruanas mencionadas.
Revelación diferenciada de juicios para la aplicación de políticas contables y de juicios para las estimaciones contables críticas

Se identificó que siete empresas mineras peruanas presentaron una revelación diferenciada de juicios. La Tabla 2 presenta las empresas que revelan por separado estos juicios y el detalle a los que hacen referencia.

En cuanto a las políticas contables que tienen efecto en la medición de los diferentes hechos económicos, se identificó que los juicios hacen referencia a los criterios y análisis. Por su parte, la Tabla 3 presenta a las empresas que revelan de manera conjunta ambos juicios, bajo la denominación general de supuestos y estimaciones contables críticas.

Como resultado del análisis comparativo de las tablas 2 y 3, se identificó conceptos que algunas empresas consideran indistintamente como ambos tipos de juicios, por ejemplo: determinación de la moneda funcional, reconocimiento y medición de provisiones por contingencias, definición de vida útil, entre otros. Esto muestra que los criterios de revelación diferenciada de juicios no se cumplen a cabalidad, lo que podría generar error de interpretación e impacto en la evaluación de dichas empresas.

\section{Descripción de nivel de revelación de supuestos asumidos en juicios y su cumplimiento en relación con las NIIF}

El principal juicio revelado como política contable es la provisión por contingencias y su probabilidad de ocurrencia asociada, mientras que aquel juicio revelado por estimaciones contables críticas es la provisión para remediación y cierre de minas. Con respecto al análisis de contingencias y probabilidad de ocurrencia, se parte de los siguientes aspectos relevantes tanto de la NIC 1 como de la NIC 8:

- Considerar las exigencias de la NIC 37 Provisiones, pasivos contingentes $y$ activos contingentes, en lo referente al concepto de contingencia y sus efectos.

Tabla 1

Cantidad de conceptos revelados como juicios, año 2020

\begin{tabular}{lc}
\hline \multicolumn{1}{c}{ Concepto } & Año 2020 \\
\hline Juicios en la aplicación de políticas contables & 25 \\
Juicios en las estimaciones contables críticas & 79 \\
\hline Fuente: Elaboración propia sobre la base de los estados financieros de las once empresas mineras peruanas
\end{tabular}


Tabla 2

Empresas mineras que presentan por separado los juicios, año 2020

\begin{tabular}{|c|c|c|}
\hline Empresa & $\begin{array}{l}\text { Juicios en la aplicación de políticas } \\
\text { contables }\end{array}$ & $\begin{array}{c}\text { Juicios en las estimaciones contables } \\
\text { críticas }\end{array}$ \\
\hline $\begin{array}{l}\text { Buenaventura } \\
(2020)\end{array}$ & $\begin{array}{l}\text { Contingencias y probabilidad de ocurrencia } \\
\text { Inicio de etapa de desarrollo } \\
\text { Inicio de etapa de producción } \\
\text { Definición de vida útil }\end{array}$ & $\begin{array}{l}\text { Estimación de reservas de mineral } \\
\text { Método de unidades de producción } \\
\text { Provisión para remediación y cierre de minas } \\
\text { Inventarios (VNR) } \\
\text { Deterioro de activos no financieros } \\
\text { Recuperabilidad del activo por impuesto a las ganancias diferido } \\
\text { Valor razonable de contraprestación contingente }\end{array}$ \\
\hline Cerro Verde (2020) & $\begin{array}{l}\text { Contingencias y probabilidad de ocurrencia } \\
\text { Costos de desbroce }\end{array}$ & $\begin{array}{l}\text { Estimación de reservas de mineral } \\
\text { Método de unidades de producción } \\
\text { Provisión para remediación y cierre de minas } \\
\text { Inventarios (VNR) } \\
\text { Deterioro de activos no financieros } \\
\text { Incertidumbre sobre tratamiento de impuesto a las ganancias }\end{array}$ \\
\hline El Brocal (2020) & $\begin{array}{l}\text { Contingencias y probabilidad de ocurrencia } \\
\text { Inicio de etapa de producción } \\
\text { Definición de vida útil }\end{array}$ & $\begin{array}{l}\text { Estimación de reservas de mineral } \\
\text { Método de unidades de producción } \\
\text { Provisión para remediación y cierre de minas } \\
\text { Inventarios (VNR) } \\
\text { Deterioro de activos no financieros } \\
\text { Recuperabilidad del activo por impuesto a las ganancias diferido } \\
\text { Incertidumbre sobre tratamiento de impuesto a las ganancias }\end{array}$ \\
\hline Minsur (2020) & $\begin{array}{l}\text { Contingencias y probabilidad de ocurrencia } \\
\text { Inicio de etapa de desarrollo } \\
\text { Costos de desbroce } \\
\text { Incertidumbre en tratamiento de impuesto a } \\
\text { las ganancias } \\
\text { Reconocimiento de inversiones } \\
\text { Reconocimiento de ingresos }\end{array}$ & $\begin{array}{l}\text { Estimación de reservas de mineral } \\
\text { Método de unidades de producción } \\
\text { Provisión para remediación y cierre de minas } \\
\text { Inventarios (VNR) } \\
\text { Deterioro de activos no financieros } \\
\text { Recuperabilidad del activo por impuesto a las ganancias diferido } \\
\text { Incertidumbre sobre tratamiento de impuesto a las ganancias } \\
\text { Determinación del costo de productos en proceso de oro } \\
\text { Estimación de la tasa de interés incremental }\end{array}$ \\
\hline Poderosa (2020) & $\begin{array}{l}\text { Definición de moneda funcional } \\
\text { Incertidumbre en tratamiento de impuesto a } \\
\text { las ganancias } \\
\text { Reconocimiento de ingresos } \\
\text { Reconocimiento de arrendamiento }\end{array}$ & $\begin{array}{l}\text { Deterioro de cuentas por cobrar } \\
\text { Estimación de reservas de mineral } \\
\text { Provisión para remediación y cierre de minas } \\
\text { Inventarios (VNR) } \\
\text { Incertidumbre sobre tratamiento de impuesto a las } \\
\text { ganancias } \\
\text { Reconocimiento y medición de provisiones por contingencias } \\
\text { Medición de los valores razonables } \\
\text { Ajustes provisionales de precios de venta } \\
\text { Adquisición de subsidiaria }\end{array}$ \\
\hline SIMSA (2020) & $\begin{array}{l}\text { Definición de moneda funcional } \\
\text { Incertidumbre en tratamiento de impuesto a } \\
\text { las ganancias } \\
\text { Reconocimiento de ingresos } \\
\text { Reconocimiento de arrendamiento } \\
\text { Reconocimiento de costos de exploración y } \\
\text { de desarrollo }\end{array}$ & $\begin{array}{l}\text { Deterioro de cuentas por cobrar } \\
\text { Deterioro de activos no financieros } \\
\text { Incertidumbre sobre tratamiento de impuesto a las ganancias } \\
\text { Definición de vida útil, valor residual y valor recuperable } \\
\text { Negocio en marcha } \\
\text { Reconocimiento y medición de provisiones por contingencias }\end{array}$ \\
\hline Southern (2020) & Consolidación de subsidiarias & $\begin{array}{l}\text { Provisión para remediación y cierre de minas } \\
\text { Deterioro de activos no financieros } \\
\text { Incertidumbre sobre tratamiento de impuesto a las ganancias } \\
\text { Definición de vida útil, valor residual y valor recuperable } \\
\text { Reconocimiento y medición de provisiones por contingencias } \\
\text { Determinación de moneda funcional } \\
\text { Estimación de mineral recuperable de los depósitos de material } \\
\text { lixiviable } \\
\text { Capitalización de costos de desbroce }\end{array}$ \\
\hline
\end{tabular}

Fuente: Elaboración propia sobre la base de los estados financieros de Compañía de Minas Buenaventura S.A.A. (2020), Sociedad Minera Cerro Verde S.A.A. (2020), Sociedad Mineral El Brocal S.A.A. (2020), Minsur S.A. (2020), Compañía Minera Poderosa S.A. (2020), Compañía Minera San Ignacio de Morococha S.A.A. (2020) y Southern Perú Copper Corporation, Sucursal del Perú (2020) 
Tabla 3

Empresas mineras que presentan conjuntamente los juicios, año 2020

\begin{tabular}{|c|c|}
\hline Empresa & Supuestos y estimaciones contables críticas \\
\hline Corona (2020) & $\begin{array}{l}\text { Estimación de reservas de mineral } \\
\text { Provisión para remediación y cierre de minas } \\
\text { Deterioro de activos no financieros } \\
\text { Incertidumbre sobre tratamiento de impuesto a las ganancias } \\
\text { Recuperabilidad de activos de exploración y evaluación }\end{array}$ \\
\hline Nexa Atacocha (2020) & $\begin{array}{l}\text { Provisión para remediación y cierre de minas } \\
\text { Deterioro de activos no financieros } \\
\text { Incertidumbre sobre tratamiento de impuesto a las ganancias } \\
\text { Estimación del valor razonable de los instrumentos financieros } \\
\text { Estimación de incertidumbres en relación con arrendamientos }\end{array}$ \\
\hline Nexa Perú (2020) & $\begin{array}{l}\text { Estimación de reservas de mineral } \\
\text { Provisión para remediación y cierre de minas } \\
\text { Deterioro de activos no financieros } \\
\text { Incertidumbre sobre tratamiento de impuesto a las ganancias } \\
\text { Estimación del valor razonable de los instrumentos financieros } \\
\text { Vida útil, valor residual y recuperable de derechos mineros, costo de desarrollo, propiedades, planta } \\
\text { y equipo, y activos intangibles }\end{array}$ \\
\hline Volcan (2020) & $\begin{array}{l}\text { Estimación de reservas de mineral } \\
\text { Provisión para remediación y cierre de minas } \\
\text { Inventarios (VNR) } \\
\text { Deterioro de activos no financieros } \\
\text { Incertidumbre sobre tratamiento de impuesto a las ganancias } \\
\text { Estimación del valor razonable de los instrumentos financieros } \\
\text { Reconocimiento y medición de provisiones por contingencias } \\
\text { Determinación de moneda funcional } \\
\text { Vida útil, valor residual y recuperable de derechos mineros, costo de desarrollo, propiedades, planta } \\
\text { y equipo, y activos intangibles } \\
\text { Reconocimiento de ingresos } \\
\text { Medición de activos mantenidos para la venta }\end{array}$ \\
\hline
\end{tabular}

Fuente: Elaboración propia sobre la base de los estados financieros de Sociedad Minera Corona S.A. (2020), Nexa Resources Atacocha S.A.A. (2020), Nexa Resources Perú S.A.A. (2020) y Volcan Compañía Minera S.A.A. (2020)

- Presentar la información relevante que muestre el fondo económico de las contingencias y los criterios de evaluación de probabilidad de ocurrencia.

- Describir los criterios de reconocimiento y medición de las contingencias y su reflejo en los estados financieros (como provisión o revelación).

Un resumen de dicho análisis para aquellas empresas mineras peruanas que lo revelaron como política contable se presenta en la Tabla 4.

Como resultado, las cuatro empresas mineras peruanas:

- Revelaron que la gerencia realizó juicios para la evaluación de contingencias de acuerdo con la NIC 37 de manera genérica.

- Efectuaron una revelación genérica respecto de la resolución de contingencias que responde a eventos futuros y su ocurrencia, uso de estimados sobre su resultado, así como la opinión de especialistas y experiencia de la gerencia.

- Finalmente, no revelaron información específica sobre los supuestos asumidos en el reconocimiento y medición de las contingencias.

Del mismo modo, se realizó el análisis de contenido sobre información respecto de dichos juicios para el caso de la provisión y remediación de cierre de minas. Para ello, se consideraron los aspectos relevantes de la NIC 1 en relación con los siguientes supuestos asumidos:

- La naturaleza de la incertidumbre en la estimación, a efectos de conocer las fuentes que la originan.

- La sensibilidad del valor registrado y las causas que lo expliquen, a fin de identificar la medida del impacto de un cambio en los supuestos asumidos. 
- La determinación esperada de la incertidumbre asociada, a efectos de conocer las expectativas de la gerencia hacia el futuro.

- $\quad$ El rango de efectos razonablemente aceptables dentro del próximo año, a fin de comprender cuál sería el impacto razonable en el siguiente periodo.

- La descripción de los cambios realizados a los supuestos definidos anteriormente, con el fin de identificar si se produjeron cambios en los supuestos asumidos.

Un resumen de dicho análisis se presenta en la Tabla 5.

De esa manera, se identificó que las empresas mineras peruanas:

- Revelaron de manera general que los factores a considerar para la incertidumbre en la medición y sus fuentes involucran cambios en tecnología, regulación, flujos, tasas de inflación y de descuento, así como en los plazos.

- En cuanto a la sensibilidad del valor registrado y las causas que lo expliquen, solo Southern hizo una revelación parcial referente a la fecha estimada de agotamiento de reservas y el inicio de actividades de cierre.

- Solo indicaron que la provisión reconocida en los estados financieros es el mejor estimado del valor actual de los flujos futuros descontados para el cierre de mina.

- Efectuaron una revelación genérica sobre la incertidumbre de los desembolsos futuros. Solo Southern precisó el impacto de cambios en los supuestos de precios y costos sobre las reservas estimadas.

- Finalmente, solo Volcan realizó una descripción de los cambios realizados a los supuestos definidos anteriormente.

Como resultado de lo anterior, se identificó que el nivel de detalle de los supuestos asumidos no es suficiente ni específico, lo cual podría impactar en los estados financieros en su conjunto a nivel de su relevancia y representación fiel, y esto, a su vez, a nivel de valuación de empresas y toma de decisiones por parte de los usuarios.

Tabla 4

Análisis de juicios revelados en la aplicación de políticas contables: Contingencias y probabilidad de ocurrencia, año 2020

\begin{tabular}{lcccc}
\hline \multicolumn{1}{c}{ Criterios de análisis } & Buenaventura & Cerro Verde & El Brocal & Minsur \\
\hline $\begin{array}{l}\text { Supuestos asumidos por requerimientos de la NIC 37: concepto } \\
\text { y efectos }\end{array}$ & Genérica & Genérica & Genérica & Genérica \\
$\begin{array}{l}\text { Supuestos asumidos en la información relevante sobre el fondo } \\
\text { económico y criterios de evaluación de probabilidad de ocurrencia }\end{array}$ & Genérica & Genérica & Genérica & Genérica \\
\begin{tabular}{l} 
Supuestos asumidos en el reconocimiento y medición \\
\hline
\end{tabular} & No & No & No & No \\
\hline
\end{tabular}

Fuente: Elaboración propia sobre la base de los estados financieros de Compañía de Minas Buenaventura S.A.A. (2020), Sociedad Minera Cerro Verde S.A.A. (2020), Sociedad Mineral El Brocal S.A.A. (2020), Minsur S.A. (2020)

Tabla 5

Análisis de juicios revelados en las estimaciones contables críticas: Provisión y remediación de cierre de minas, año 2020

\begin{tabular}{l}
\hline Criterios de análisis \\
\hline Naturaleza de la incertidumbre en la estimación y sus fuentes \\
Sensibilidad del valor registrado y las causas que lo expliquen que permita medir el impacto de un cambio (1) \\
Determinación esperada de la incertidumbre que permita conocer la expectativa de la empresa \\
Rango de efectos razonablemente aceptables dentro del próximo año que permitan comprender el impacto razonable (1) Genérica \\
Descripción de los cambios realizados a los supuestos definidos anteriormente (2) No
\end{tabular}

Nota: (1) Revelación parcial de Southern Perú Copper Corporation, Sucursal del Perú (2020) y (2) Mayor referencia en la revelación de Volcan Compañía Minera S.A.A. (2020)

Fuente: Elaboración propia 


\section{DISCUSIÓN}

La discusión de resultados se desarrolla de acuerdo con los objetivos planteados. En primer lugar, cuatro de las once empresas mineras peruanas no efectuaron una revelación diferenciada de los juicios. Al respecto, Chiahemba e Ibiamke (2019) confirman esta situación en su investigación desarrollada en Nigeria. Mientras que si bien Burcă et al. (2019) y Albrecht et al. (2017) también coinciden que tales resultados podrían afectar la valuación de las empresas, esta investigación detalla los juicios revelados por las empresas mineras peruanas. En segundo lugar, la revelación asociada a estos juicios es genérica en su mayoría y no proporciona mayor detalle sobre los supuestos asumidos por la gerencia, lo cual se confirma con el estudio elaborado por Ernst and Young (2017) (como se citó en Chiahemba e Ibiamke, 2019). En el caso de Raubenheimer (2013), este fue solo enunciativo, mientras que la presente investigación realiza un análisis del contenido de las estimaciones contables críticas de las empresas mineras peruanas en función de las exigencias expuestas de la NIC 1 y la NIC 8.

En conclusión, respecto de la identificación de una revelación diferenciada de los juicios, la ausencia de una clara distinción entre juicios ocasiona una interpretación errónea de la información incluida en los estados financieros, lo que muestra una apertura de criterios en cuanto a su diferenciación, reflejando una mayor preferencia por su impacto en los resultados y posición financiera, lo que afectaría decisiones de desempeño y de negocio. Como recomendación, es necesario realizar investigaciones que profundicen estos aspectos para su adecuado tratamiento contable.

Por otra parte, con relación al nivel de revelación de los supuestos asumidos, dicha revelación es genérica y estandarizada a nivel de contenido en las notas, generando una información poco confiable sobre el tipo de gestión, el valor de la empresa y los criterios que asume la gerencia respecto del cumplimiento del marco normativo de las NIIF. Como recomendación es necesario fijar criterios de soporte a los juicios ejercidos en la preparación de estados financieros.

El aporte de la presente investigación se focaliza en el análisis del cumplimiento del requerimiento de las NIIF respecto de la revelación de información de los juicios, lo que contribuye a un mejor reflejo de su tratamiento contable. La agenda para futuras investigaciones debería dar respuesta a las siguientes preguntas: ¿Qué tipo de criterios específicos estandarizados deberían definirse para los supuestos asumidos por la gerencia?; ¿a qué nivel de detalle deberían definirse las exigencias de cumplimiento en la revelación? y ¿a qué otros sectores económicos se podría dar el análisis desarrollado en la presente investigación?

\section{REFERENCIAS}

Albrecht, A., Kim, K., \& Lee, K. (2017). Changes in Accounting Estimates: Are the Current Disclosure Requirements Sufficient to Deter Managerial Opportunism? KAIST College of Business Working Paper Series, (22). https://dx.doi.org/10.2139/ssrn.3074071

Burcă, V., Nicolăescu, \& C. Drăgut, D. (2019). Critical Analysis on the Amendments Discussed, Concerning Changes in Accounting Estimates. Studies in Business and economics, 14(1), 17-33. https://doi.org/10.2478/ sbe-2019-0002

Chiahemba, C., \& Ibiamke, A. (2019). Disclosure of Critical Accounting Judgments and Key Sources of Estimation Uncertainty in the Financial Statements of Companies in Nigeria. Journal of Finance and Accounting, 7(1), 22-31. https://doi.org/10.11648/j.jfa.20190701.14

Compañía de Minas Buenaventura S.A.A. (2020). Estados financieros al 31 de diciembre de 2020 y 2019 junto con el dictamen de auditores independientes. Recuperado del sitio de Internet de la Superintendencia de Mercado de Valores: https://www.smv.gob.pe/ConsultasP8/ temp/Informe\%20Anual\%20Separado.pdf

Compañía Minera Poderosa S.A. (2020). Estados financieros separados 31 de diciembre de 2020 y de 2019 (con el dictamen de auditores independientes). Recuperado del sitio de Internet de la Superintendencia de Mercado de Valores: https://www.smv.gob.pe/ConsultasP8/ temp/EEFF\%20Individuales\%20Auditados\%20Pode$\underline{\text { rosa } \% 202020 . p d f}$

Compañía Minera San Ignacio de Morococha S.A.A. (2020). Estados financieros separados 31 de diciembre de 2020 y de 2019 (con el dictamen de auditores independientes). Recuperado del sitio de Internet de la Superintendencia de Mercado de Valores: https://www. smv.gob.pe/ConsultasP8/temp/EEFF\%20SIMSA\%20 2020.pdf

Glendening, M. R. (2012). Critical accounting estimate disclosure and the value relevance of balance sheets items (Tesis doctoral, Universidad de Iowa, Estados Unidos de América). https://doi.org/10.17077/etd.6xgulnh

Hendriksen, E., \& van Breda, M. (1992). Accounting

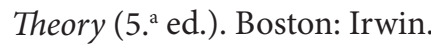

International Financial Reporting Standards Foundation. (2020). Normas NIIF Parte A, Parte B y Parte C. Normas emitidas al 1 de enero de 2020. Recuperado de https://www.ifrs.org/ 
Michels, J. (2017). Disclosure versus Recognition: Inferences from Subsequent Events. Journal of Accounting Research, 55(1), 3-34. https://doi.org/10.1111/1475-679X.12128

Minsur S.A. (2020). Estados financieros separados al 31 de diciembre de 2020 y de 2019 junto con el dictamen de auditores independientes. Recuperado del sitio de Internet de la Superintendencia de Mercado de Valores: https://www.smv.gob.pe/ConsultasP8/temp/Auditado\%20Minsur\%20Separado\%202020.pdf

Nexa Resources Atacocha S.A.A. (2020). Estados financieros individuales al 31 de diciembre de 2020 y dictamen de auditores independientes. Recuperado del sitio de Internet de la Superintendencia de Mercado de Valores: https://www.smv.gob.pe/ConsultasP8/temp/0922NRAtacochaIndiv20.pdf

Nexa Resources Perú S.A.A. (2020). Estados financieros al 31 de diciembre de 2020 y 2019 junto con el dictamen de auditores independientes. Recuperado del sitio de Internet de la Superintendencia de Mercado de Valores: https://www.smv.gob.pe/ConsultasP8/temp/0922NRPeruSEP20.pdf

Raubenheimer, E. (2013). Accounting Estimates in Financial Statements and their Disclosure by some South African Construction Companies. Journal of Economic and Financial Sciences, 6(2), 383-400. Recuperado de https://jefjournal.org.za/index.php/jef/article/download/266/346

Sociedad Minera Cerro Verde S.A.A. (2020). Estados financieros al 31 de diciembre de 2020 y de 2019 junto con el dictamen de auditores independientes. Recuperado del sitio de Internet de la Superintendencia de Mercado de Valores: https://www.smv.gob.pe/ConsultasP8/temp/Informe\%20Cerro\%20Verde\%2031\%20 12\%2020\%2019\%20Espa\%c3\%b1ol.pdf
Sociedad Minera Corona S.A. (2020). Estados financieros al 31 de diciembre de 2020 y 2019 junto con el dictamen de auditores independientes. Recuperado del sitio de Internet de la Superintendencia de Mercado de Valores: https://www.smv.gob.pe/ConsultasP8/temp/ EEFF\%20Auditados Corona\%202020.pdf

Sociedad Mineral El Brocal S.A.A. (2020). Estados financieros al 31 de diciembre de 2020 y 2019 junto con el dictamen de auditores independientes. Recuperado del sitio de Internet de la Superintendencia de Mercado de Valores: https://www.smv.gob.pe/ConsultasP8/temp/ SMEB2020.pdf

Southern Perú Copper Corporation, Sucursal del Perú. (2020). Estados financieros al 31 de diciembre de 2020 y 2019 junto con el dictamen de auditores independientes. Recuperado del sitio de Internet de la Superintendencia de Mercado de Valores: https://www.smv.gob.pe/ ConsultasP8/temp/SPCC\%20EEFF\%20Aud\%202020. pdf

Volcan Compañía Minera S.A.A. (2020). Estados financieros al 31 de diciembre de 2020 y 2019 junto con el dictamen de auditores independientes. Recuperado del sitio de Internet de la Superintendencia de Mercado de Valores: https://www.smv.gob.pe/ConsultasP8/temp/ EEFF Sep Aud31dic20.pdf 